\title{
Innovación social: un análisis bibliométrico del concepto y sus tendencias actuales
}

\author{
Leydis Marcela Maestre Matos* \\ Andrea Paola Páez Cabas** \\ Jahir Enrique Lombana Coy*** \\ Jaider Manuel Vega Jurado****
}

Fecha de recibido: 21 de abril de 2020

Fecha de aprobado: 20 de noviembre de 2020

Para citar este artículo: Maestre Matos, L. M., Páez Cabas, A. P., Lombana Coy, J. E.,
\& Vega Jurado, J. M. (2021). Innovación social: un análisis bibliométrico del concepto
y sus tendencias actuales. Revista Universidad E Empresa, 23(41), 1-27. https://doi. y sus tendencias actuales. Revista Universidad \& Empresa, 23(41), 1-27. https://doi.
org/10.12804/revistas.urosario.edu.co/empresa/a.8964

\section{Resumen}

El concepto de innovación social se ha desarrollado desde el año 2006, por lo que es considerado un término novedoso que se encuentra en etapa de construcción. Este artículo tiene como objetivo dar a conocer el inicio, evolución y tendencias actuales de la innovación social, además de mostrar los autores seminales y con mayor número de publicaciones, las revistas con tendencias de publicación del tema, las metodologías utilizadas y los países que apoyan estas investigaciones. La metodología empleada consistió en una revisión sistemática de la literatura a través del análisis bibliométrico con aplicación de la teoría de

* Doctora en Administración, Universidad del Norte (Barranquilla, Colombia); magíster en Administración de Negocios, Universidad del Norte (Barranquilla, Colombia). Correo electrónico: matosl@uninorte.edu.co oRCID: https://orcid. org/0000-0001-5390-9478

** Ingeniera Industrial, Universidad del Magdalena (Santa Marta, Colombia). Correo electrónico: andreap94c@gmail.com ORCID: https://orcid.org/0000-0002-5681-675X

*** PhD en Economía, Universität Goettingen (Alemania). Profesor asociado de la Universidad del Norte (Barranquilla, Colombia). Correo electrónico: lombanaj@uninorte.edu.co ORCID: https://orcid.org/0000-0002-0055-8392

**** PhD en Proyectos de Ingeniería e Innovación, Universidad Politécnica de Valencia (España). Profesor asistente de la Universidad del Norte (Barranquilla, Colombia). Correo electrónico: jaiderv@uninorte.edu.co ORCID: https://orcid. org/0000-0001-9995-643X 
grafos. Dentro de los hallazgos más sobresalientes, se encontró que el concepto de innovación social es heterogéneo y sin límites definidos, y que su análisis se está generando en cuatro niveles: general, macro, meso y micro, cada uno con sus tendencias internas. Sin embargo, se presentan aún deficiencias metodológicas, relacionadas con marcos teóricos para la investigación del concepto y ausencia de estudios sectoriales con casos específicos que evidencien la aplicación de la innovación social.

Palabras clave: innovación social; análisis bibliométrico; tendencias conceptuales; teoría de grafos; tree of science.

\title{
Social Innovation: A Bibliometric Analysis of the Concept and its Current Trends
}

\begin{abstract}
The social innovation concept was developed in 2006, which is why it is considered a new term in the construction stage. This article aims to present the beginning, evolution, and current trends of social innovation, in addition to showing the seminal authors and those with the highest number of publications, journals with publication trends on the subject, the methodologies used, and the countries that support this research. The methodology consisted of a literature systematic review through its bibliometric analysis with the application of graph theory. Among the most outstanding findings is that the concept is heterogeneous without defined limits. Its analysis was generated at four levels: general, macro, meso, and micro, each with its internal tendencies. However, there are still methodological deficiencies related to theoretical frameworks for the concept of investigation and the absence of sectorial studies with specific cases that show the application of social innovation.
\end{abstract}

Keywords: Social innovation; bibliometric analysis; conceptual trends; graph theory; tree of science.

\section{Inovação social: uma análise bibliométrica do conceito e suas tendências atuais}

\section{Resumo}

O conceito de inovação social vem sendo desenvolvido desde 2006, por isso é considerado um novo termo que está em fase de construção. Este artigo tem como objetivo apresentar o início, a evolução e as tendências atuais da inovação social, além de mostrar os autores seminais e com maior número de publicações, os periódicos com tendências de publicação sobre o assunto, as metodologias utilizadas e os países que dão suporte a essas investigações. A metodologia utilizada consistiu em uma revisão sistemática da literatura por meio da análise bibliométrica com a aplicação da teoria de grafos. Dentre os achados mais destacados, constatou-se que o conceito de inovação social é heterogêneo e sem limites definidos e que sua análise está sendo gerada em quatro níveis: geral, macro, meso e micro, cada um com suas tendências internas. No entanto, ainda existem deficiências metodológicas, relacionadas aos referenciais teóricos para a investigação do conceito e a ausência de estudos setoriais com casos específicos que evidenciam a aplicação da inovação social.

Palavras-chave: inovação social; análise bibliométrica; tendências conceituais; teoria de grafos; tree of science. 


\section{Introducción}

Durante el siglo xxi alrededor del mundo se ha venido consolidando el concepto y la práctica de la innovación en diferentes escenarios: científico, tecnológico, económico, político, cultural y social, los cuales han transformado gradualmente los procesos de desarrollo de los territorios, las industrias y las personas inmersas en una comunidad determinada (Arcos Soto, Suárez Pineda \& Zambrano Vargas, 2015).

En la investigación desarrollada por Martins, Rindova y Greenbaum (2015) se presenta la innovación como un proceso de creación de valor que puede darse desde el razonamiento analógico o desde la combinación conceptual. Para Meissner y Kotsemir (2016), la esencia de la innovación está en el conocimiento y las ideas que dan lugar a nuevas soluciones en los ámbitos: tecnológico, económico, organizacional y social.

En este sentido, Souto (2015) identifica tres tipos principales de innovación: innovación de productos, innovación de procesos e innovación organizativa, las cuales coinciden con la clasificación propuesta por Fichman, Dos Santos y Zheng (2014), con la diferencia de que este último en lugar de una innovación organizativa plantea la innovación del modelo comercial. Además, es posible encontrar en la literatura trabajos relacionados con innovación tecnológica (Stephan, Schmidt, Bening \& Hoffmann, 2017; Silva, Styles \& Lages, 2017), innovación de servicios (Cheng \& Krumwiede, 2017), innovación de mercado (Silva et al., 2017) e innovación social (Edwards-Schachter \& Wallace, 2017; Van der Have \& Rubalcaba, 2016; Phillips, Lee, Ghobadian, O'Regan \& James, 2015; Cajaiba Santana, 2014). Autores como Cajaiba Santana (2014) y Phillips et al. (2015) destacan el creciente interés de los investigadores en el estudio de este último tipo de innovación (social) y la existencia de brechas de conocimiento que aún pueden ser trabajadas en este tema.

La aplicación de la innovación social en los trabajos desarrollados por universidades (Stanford, Québec, Duke y Harvard) y algunas organizaciones como Nesta, la Comisión Europea, el Centro de Innovación Social del País Vasco (Lehtola \& Ståhle, 2014) ha hecho que este término tome relevancia tanto en investigaciones académicas como prácticas. 
Autores como Mulgan (2006), Mulgan, Tucker, Ali y Sanders (2007), Moulaert, Martinelli, González y Swyngedouw (2007) y Phills, Deiglmeier y Miller (2008) han aportado definiciones al concepto de innovación social que se constituyen en referentes clave para los actuales investigadores que estudian el tema. Sin embargo, muchos de ellos coinciden en que el concepto de innovación social es amplio y aún no ha llegado a un consenso definitivo en cuanto a su alcance y operacionalización (Bonifacio, 2014; Sanzo Pérez, Álvarez González \& Rey García, 2015; Klein, 2013; Howaldt \& Schwarz, 2011, Bulut, Eren \& Halac, 2013), siendo necesario el desarrollo de revisiones de literatura que contribuyan a darle claridad.

Algunas revisiones de literatura han estudiado el uso del concepto de innovación social (Edwards-Schachter \& Wallace, 2017). Van der Have y Rubalcaba (2016), por su parte, identifican el comportamiento de la producción científica en innovación social a lo largo del tiempo y establecen conglomerados con los artículos estrechamente relacionados; y Phillips et al. (2015) aportan una visión general de las investigaciones que relacionan la innovación social con el emprendimiento social.

Este artículo, por su parte, brinda información actualizada a 2018 y utiliza el análisis bibliométrico de los artículos para establecer una línea de tiempo desde los primeros conceptos de innovación social hasta sus tendencias actuales, mostrando autores seminales y autores con mayor número de publicaciones; revistas con tendencias de publicación del tema; países que apoyan el desarrollo de estas investigaciones y las principales metodologías empleadas para el análisis de la innovación social, evidenciando un panorama amplio de las condiciones actuales en la materia y las potenciales líneas de investigación.

Por lo anterior, este artículo responde diferentes preguntas de investigación: ¿cuáles son los inicios y tendencias actuales de la innovación social? ¿Quiénes son los autores seminales y los autores con mayor número de publicaciones en innovación social? ¿Cuáles son las principales metodologías utilizadas y los países que más apoyan el desarrollo de investigaciones en innovación social?

La investigación realizada se muestra en el presente artículo con la siguiente estructura: en la próxima sección se describe de forma amplia la metodología usada, continuando con los hallazgos encontrados, que incluyen el análisis estadístico de las publicaciones sobre innovación social, los métodos utilizados para la investigación sobre el tema, la 
aproximación al concepto y sus tendencias de investigación. Y, para culminar, se presentan las discusiones y conclusiones encontradas en la revisión de la literatura.

\section{Metodología}

Una vez identificado el tema y las preguntas de investigación, se procedió a aplicar la teoría de grafos (Euler, 1741) para la conducción de la revisión de la literatura, la cual consiste en identificar los artículos pertinentes al respecto y, a través del análisis de las propiedades de redes estructurales y los indicadores del grado de entrada y salida de los nodos de la red (basado en el número citaciones del artículo), se clasifican los artículos en seminales o tendenciales.

Aunque aún no hay una claridad del concepto de redes de conocimiento (Liu, Jiang \& Ma, 2013), se considera que los nodos representan unidades de conocimiento (artículos) y los enlaces indican las conexiones entre estos artículos (referencias de los artículos) (Robledo Giraldo, Duque Méndez \& Zuluaga Giraldo, 2013). Dicha representación se muestra en un gráfico que se ha denominado tree of science (árbol de la ciencia) y su distinción depende del grado de entrada y salida en los nodos de la red. Por lo tanto, las raíces son artículos con grado de entrada alto y salida cero; el tronco, artículos con un grado de intermediación alto; y las hojas del árbol, artículos que tienen un grado de salida alto y un grado de entrada cero. Las raíces representan los autores principales y de origen del concepto; el tronco, los autores que han aportado a la evolución de este; las ramas con sus hojas son las perspectivas (tendencias) y los autores que han aportado a dichas perspectivas (Robledo, Osorio \& López, 2014).

\subsection{Búsqueda de la información}

Teniendo en cuenta el procedimiento establecido para el análisis bibliométrico con el tree of science, se desarrolló la búsqueda utilizando la base de datos Thomson Reuters' Web of Science (WoS) en todos los períodos y refinado por artículos, usando la siguiente búsqueda: Ts: (social innovation). Período: todos los años. Tipo de documentos: artículos. 
Índices: sCI-Expanded, ssCI, A\&HCI, ESCI. Refinados por las cinco primeras áreas que aparecen en la categorización: management, business, environmental studies, social sciences interdisciplinary y economics.

La búsqueda arrojada por el WoS fue de 471 artículos y fue utilizada para el análisis con la teoría de grafos desarrollada por Robledo Giraldo et al. (2013). Esta aplicación permitió obtener una red de 471 artículos con 980 citaciones, resaltando 80 artículos como los más citados en el tema.

\subsection{Organización y análisis de la información}

Una vez identificados los artículos con mayor número de citaciones, se realizó una lectura crítica de cada uno de ellos, cuyo análisis de contenido fue organizado en una matriz diseñada en el programa Excel 2016, que incluyó ítems de identificación del artículo (autores, revista, fecha de publicación, país de procedencia de la investigación), clasificación según la teoría de grafos (seminales o tendencial), palabras clave, metodología (tipo de metodología aplicada), concepto(s) de innovación social empleado en la investigación, tema de investigación, conclusiones y observaciones relevantes del artículo.

Esta organización de la información permitió hacer análisis estadístico (tablas y gráficos) de datos, identificación del concepto y la utilización de conceptos ya existentes por otros autores, así como la clasificación de las tendencias de investigación según niveles (general, macro, meso y micro), de acuerdo con el tema de los artículos y las conclusiones establecidas. Los resultados de los análisis se muestran en la siguiente sección.

\section{Resultados}

En esta sección se presentan los hallazgos de la revisión de la literatura en innovación social. En primer lugar, se ofrece un análisis estadístico de las publicaciones de innovación social, en las que se destacan principales autores, revistas, países y palabras clave relacionadas con la investigación en Is. En segundo lugar, se describen los métodos más utilizados para abordar los 
estudios en is. En tercer lugar, se destacan los autores seminales y su aproximación conceptual a la Is. Y, finalmente, se describen las tendencias actuales en la investigación de la innovación social.

\subsection{Análisis estadístico de publicaciones de innovación social}

Hasta diciembre de 2018 se han publicado en Web of Science 471 artículos sobre innovación social, en los cuales se identificaron 162 autores que han desarrollado el tema en sus investigaciones. Teniendo en cuenta el análisis efectuado, se tiene que los autores con más publicaciones sobre innovación social son Frances R. Westley con 6 artículos y Per Olsson con 4. En la figura 1 se ilustran los primeros diez autores que produjeron el $7 \%$ de los artículos en estudio.

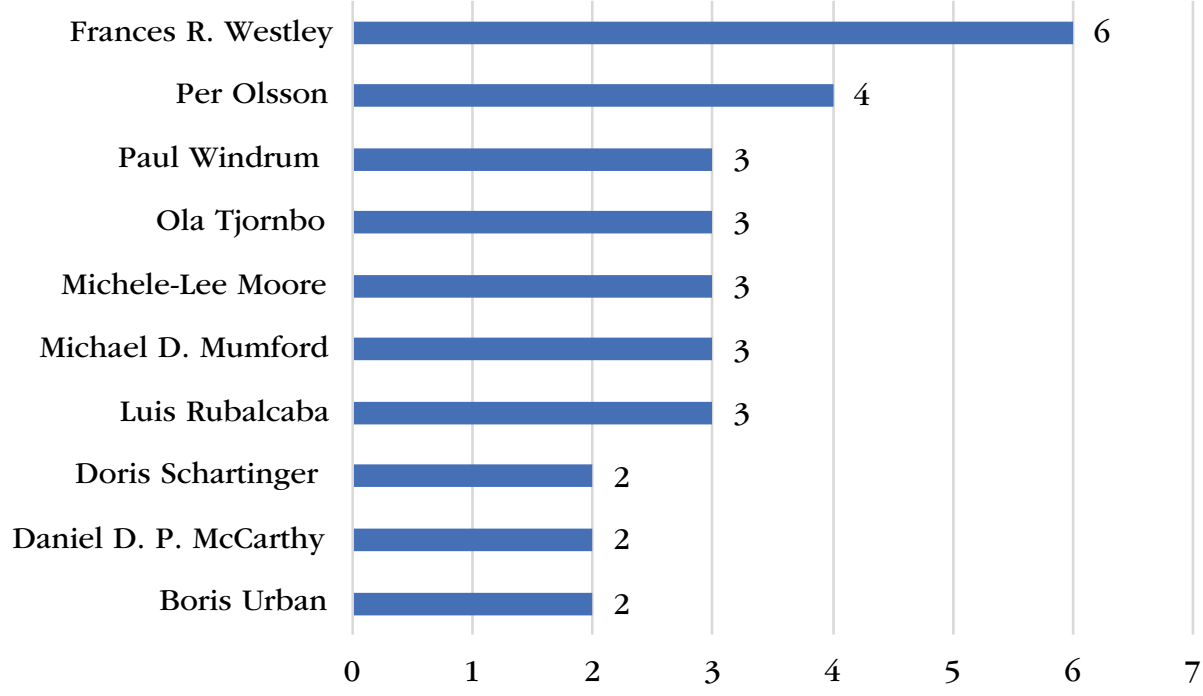

Figura 1. Principales autores que han desarrollado investigaciones en innovación social hasta 2018 Fuente: elaboración de los autores en Excel 2016 a partir de datos de Web of Science.

Los estudios sobre innovación social desarrollados en este mismo período han sido publicados en 42 revistas indexadas en WoS. Con base en los resultados, se tiene que las principales revistas (figura 2) donde más se han publicado artículos referentes a innovación social, equivalentes al 7\% de la producción científica en innovación social, son: Ecology and Society, Technological Forecasting and Social Change, European Journal of Innovation Management, Journal of Social Entrepreneurship, Industry and Innovation y Environment and Planning C. 


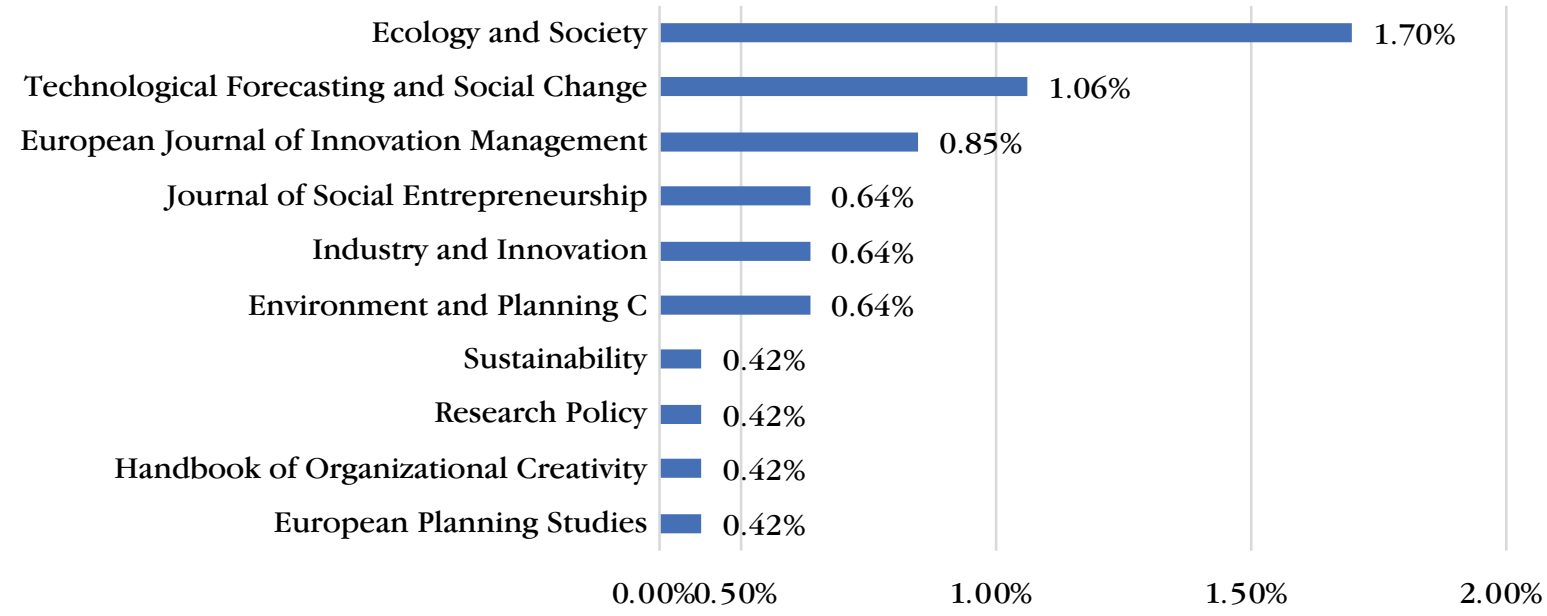

Figura 2. Principales revistas que han publicado artículos sobre innovación social hasta 2018 Fuente: elaboración de los autores en Excel 2016 a partir de datos de Web of Science.

Haciendo un análisis de las afiliaciones de cada autor, se determinaron los países de origen de los documentos. Se identificaron en total 23 naciones con producción científica en Web of Science sobre is hasta 2018. Para efectos del análisis, en la figura 3 se muestran los primeros diez:

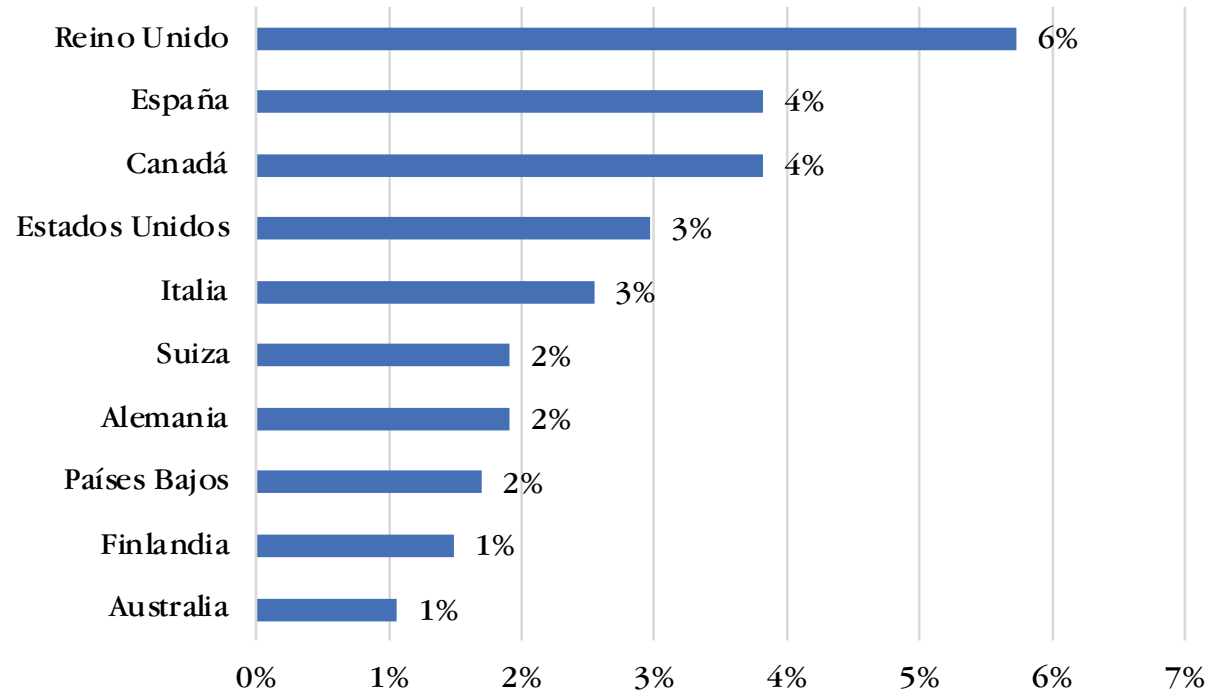

Figura 3. Principales países de origen de los documentos sobre innovación social hasta 2018 Fuente: elaboración de los autores en Excel 2016 a partir de datos de Web of Science. 
Teniendo en cuenta lo anterior, se puede inferir que el país con mayores estudios realizados sobre innovación social ha sido el Reino Unido, con el $6 \%$ de los documentos publicados, seguido de España (4\%), Canadá (4\%), Estados Unidos (3\%), Italia (3\%) y Suecia (2\%).

En cuanto a las palabras clave, se identificaron en los metadatos las palabras clave de autores y palabras clave indexadas, obteniendo así 198 palabras en los 471 documentos analizados. Para efecto del análisis, en la figura 4 se destacan las diez palabras clave más frecuentes (después de innovación social), las cuales se encuentran en el 10\% de los registros analizados:

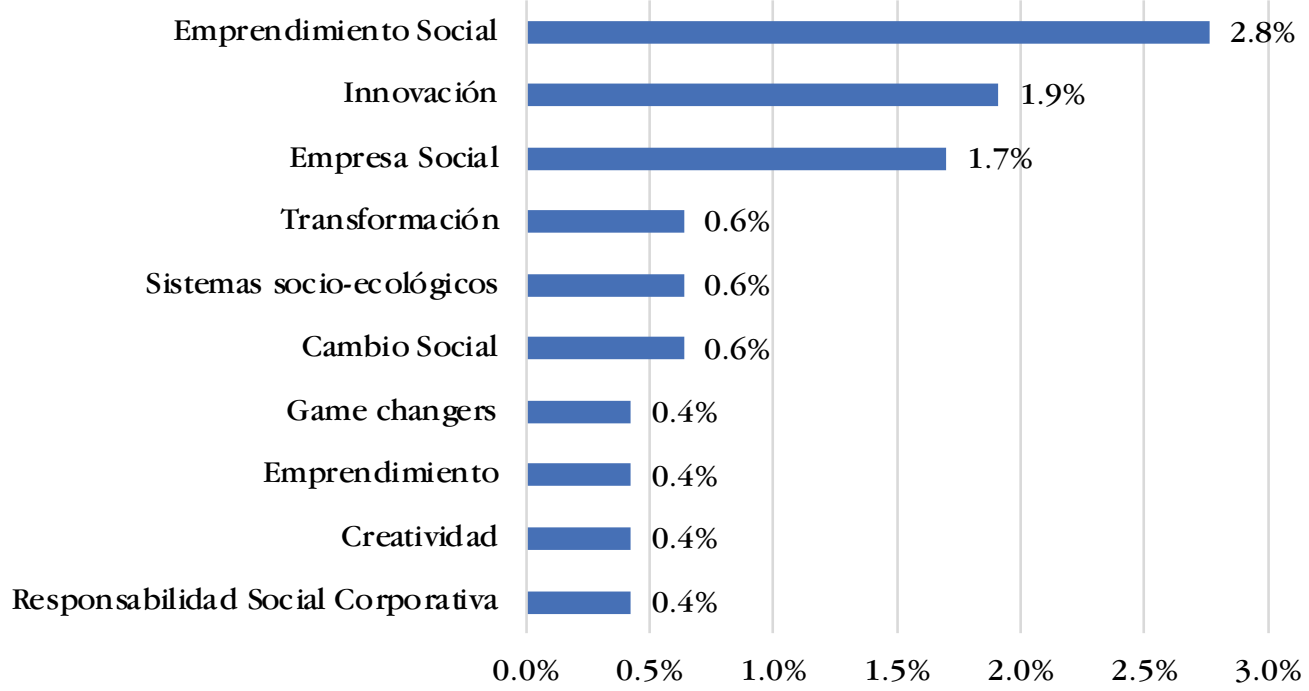

Figura 4. Principales palabras clave relacionadas con innovación social hasta 2018 Fuente: elaboración de los autores en Excel 2016 a partir de datos de Web of Science.

Por lo tanto, el concepto que más tiene relación al hablar de innovación social es emprendimiento social, estando este como palabra clave en aproximadamente el $2.8 \%$ de las publicaciones efectuadas sobre is. No obstante, se evidencian palabras clave tales como innovación, empresa social, transformación, sistemas socioecológicos y cambio social, que también muestran relación con el tema en estudio.

Cabe resaltar que, al hacer el análisis de metadatos sobre los autores que han hablado sobre innovación social, países que se destacan en la investigación del tema y palabras 
clave más utilizadas en los estudios de is, se obtuvo un amplio número de datos, lo que evidencia de igual manera que los datos no son mutuamente excluyentes, pues varios autores pueden tener filiación con un mismo documento, así como se pueden enunciar diferentes palabras clave en un solo artículo.

\subsection{Métodos utilizados para investigaciones en innovación social}

Luego de revisar la metodología de los artículos sobre innovación social, se obtuvo que el abordaje de la investigación en la temática se ha hecho a través de seis métodos principalmente. En primer lugar se encuentran las revisiones de literatura, con el $46 \%$ de los documentos; en segundo lugar, los estudios de caso (18\%); en tercer lugar están los análisis cualitativos (14\%), que incluyen etnografías, análisis de contenidos, grupos focales, análisis de observación directa, análisis de entrevistas, análisis comparativo de perfiles y análisis de discursos; en cuarto lugar están los análisis cuantitativos (11\%), que comprenden diseños experimentales, análisis de regresión y correlación, sistemas de medición de la innovación social y modelos de panel vectorial regresivo. Finalmente, se encuentran los análisis de datos secundarios (7\%) y estudios mixtos (3\%), con el quinto y sexto lugar, respectivamente (ver figura 5).

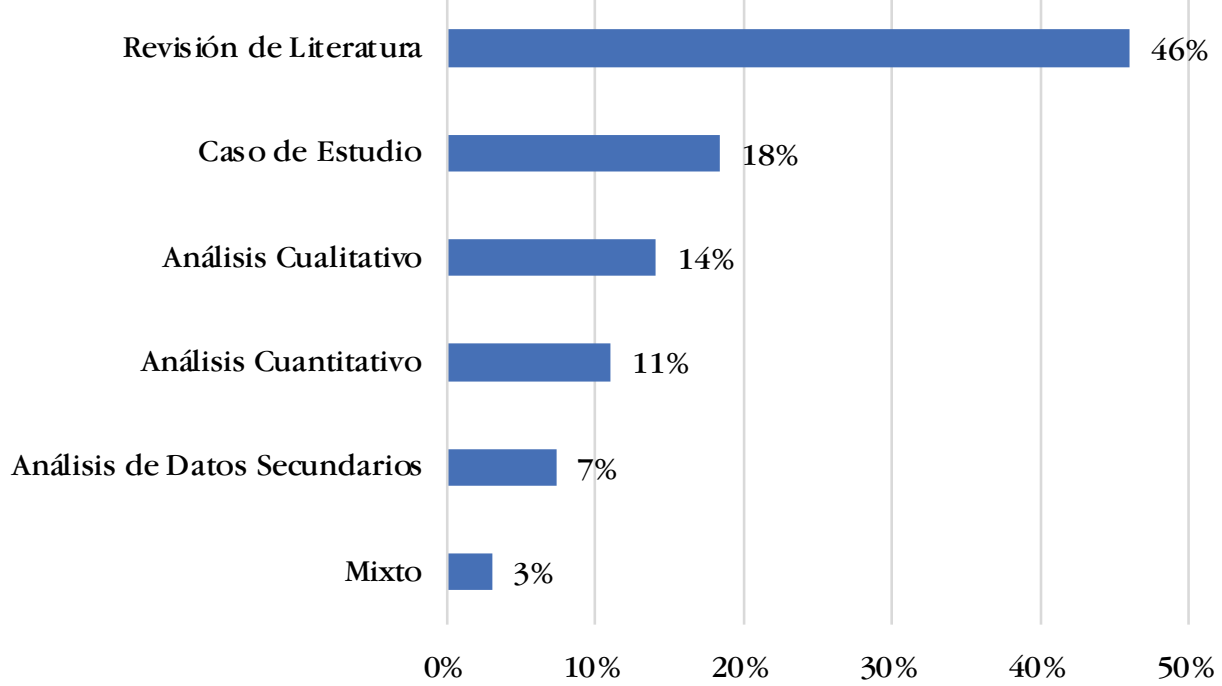

Figura 5. Métodos usados para investigación en innovación social hasta 2018

Fuente: elaboración de los autores en Excel 2016 a partir de revisión de artículos. 
Esto indica que los investigadores se han preocupado por profundizar en conocer el concepto y los documentos existentes al respecto. Así mismo, se muestra que la metodología cualitativa ha sido predominante sobre la cuantitativa, lo cual sustenta el hecho de que la is es un concepto que aún se encuentra en construcción y todavía no hay claridad sobre su medición y operacionalización, generando así oportunidades de investigación metodológica.

\subsection{Evolución del concepto de innovación social}

La construcción del concepto de innovación social se dio entre los años 2002 y 2010 con los aportes de autores como Mumford (2002), Austin, Stevenson y Wei-Skillern (2006), Mair y Martí (2006), Mulgan et al. (2007), Phills et al. (2008), Murray, Caulier-Grice y Mulgan (2009) y Howaldt y Schwarz (2010). A partir de allí se ha venido desarrollando el concepto y actualmente es posible identificar cuatro niveles de investigación: general, macro, meso y micro, que son abordados desde distintos enfoques de investigación, como se evidencia en la figura 6. 


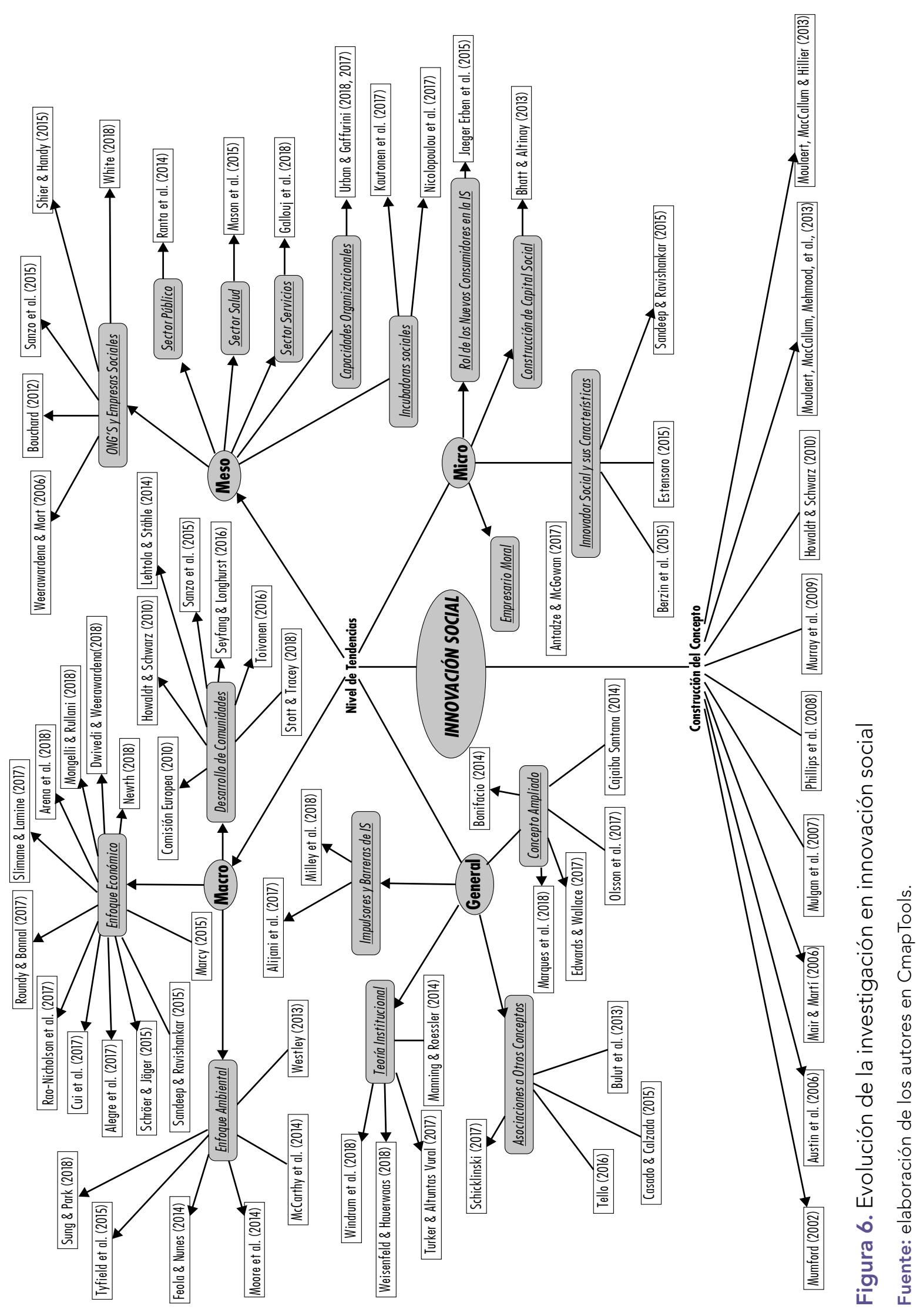




\subsubsection{Investigaciones seminales y aproximación conceptual de la innovación social}

Al referirse a innovación social, se debe recordar que la innovación puede verse como una manifestación de la capacidad creativa del ser humano y su esfuerzo para responder a sus necesidades y mejorar la calidad de vida, estando presente en todo su desarrollo histórico (Cajaiba Santana, 2014). Desde la década anterior se ha venido desarrollando fuertemente el concepto de innovación social (figura 6, ítems construcción del concepto), cuyos estudios incluyen trabajos desarrollados por universidades como Stanford, Duke, Québec y Harvard, y por organizaciones dedicadas a la innovación social, como Nesta, Zentrum für Soziale Innovation y la Fundación Joven (Lehtola \& Ståhle, 2014). No obstante, el concepto de innovación social ha sido heterogéneo y presenta límites poco definidos (Bonifacio, 2014; Sanzo Pérez et al., 2015; Klein, 2013; Howaldt \& Schwarz, 2011; Bulut et al., 2013).

El primero en hablar de innovación social fue Mumford (2002) y la definió como la generación e implementación de nuevas ideas sobre cómo las personas deben organizar las interacciones interpersonales o sociales para alcanzar los objetivos comunes. Para el año 2007, Mulgan et al. generan una mayor claridad al concepto, sosteniendo que la innovación social es el desarrollo e implementación de nuevas ideas (productos, servicios y modelos) para satisfacer las necesidades sociales; sin embargo, es explícito en afirmar que la aplicación de este concepto es predominante en organizaciones cuyos fines principales son sociales. Moulaert et al. (2007), por su parte, establecen una conexión entre innovación social y la creación de relaciones sociales; y Seyfang y Smith (2007) la vinculan al desarrollo sostenible. Phills et al. (2008) sostienen que la innovación social implica el desarrollo y la aplicación de nuevas soluciones a los problemas sociales más eficaces que las soluciones anteriores.

En el desarrollo o transición del concepto de innovación social se destacan autores como Marcy y Mumford (2007), quienes trabajan el aporte de Moulaert et al. (2007) y añaden el logro de objetivos comunes a la creación de las relaciones sociales. Witkamp, Raven y Royakkers (2011), por su parte, relacionan la innovación social con el concepto de emprendimiento social; y Neumeier (2012), con la creación de activos. 
En la tabla 1 se muestran otros conceptos desarrollados por algunos autores y la adopción de los conceptos seminales de Mulgan et al. (2007) y Phills et al. (2008) por otros autores. El concepto más reciente es el presentado por Shier y Handy (2015), que expresa que la innovación social es un modelo amplio de cambio social que pone de relieve cómo sin ánimo de lucro servicios directos pueden crear un cambio social, no solo a través del apoyo a nivel de políticas públicas, sino además dentro de sus comunidades locales y organizaciones por medio de programas innovadores y procedimientos de organización.

Tabla 1. Conceptos desarrollados de innovación social

\begin{tabular}{|c|c|}
\hline Autor & Concepto \\
\hline Mumford (2002), Marcy y Mumford (2007) & $\begin{array}{l}\text { Generación e implementación de nuevas ideas sobre cómo las } \\
\text { personas deben organizar las interacciones interpersonales o } \\
\text { sociales para alcanzar los objetivos comunes. }\end{array}$ \\
\hline $\begin{array}{l}\text { Murray et al. (2009), Moulaert, MacCallum, } \\
\text { Mehmood y Hamdouch (2013), Van Dyck y Van } \\
\text { den Broeck (2013) }\end{array}$ & $\begin{array}{l}\text { Es el tipo de innovación que permite crear o transformar nuevas } \\
\text { relaciones sociales o colaboraciones. }\end{array}$ \\
\hline Howaldt y Schwarz (2010) & $\begin{array}{l}\text { Un proceso de creación colectiva en la que los miembros de una } \\
\text { determinada unidad colectiva aprenden, inventan y diseñan nue- } \\
\text { vas reglas para el desarrollo social o, en una palabra, una nueva } \\
\text { práctica social, y en este proceso se adquieren las habilidades } \\
\text { cognitivas, racionales y organizativas necesarias. }\end{array}$ \\
\hline Neumeier (2012) & $\begin{array}{l}\text { Las innovaciones sociales son non-material: sus resultados mate- } \\
\text { riales son de forma suplementaria, no se centran en las necesida- } \\
\text { des, sino en la creación de activos. }\end{array}$ \\
\hline Klein, Fontan, Harrisson y Lévesque (2012) & $\begin{array}{l}\text { Se refiere a la implementación de nuevos acuerdos sociales e } \\
\text { institucionales, nuevas formas de movilización de recursos, nuevas } \\
\text { respuestas a los problemas para los cuales las soluciones disponi- } \\
\text { bles han demostrado aspiraciones sociales inadecuadas. }\end{array}$ \\
\hline Shier y Handy (2015) & $\begin{array}{l}\text { El modelo de innovación social es un modelo más amplio de cambio } \\
\text { social y pone de relieve cómo sin ánimo de lucro servicios directos } \\
\text { pueden crear un cambio social, no solo a través del apoyo a nivel de } \\
\text { políticas públicas, sino además dentro de sus comunidades locales y } \\
\text { organizaciones por medio de programas innovadores y procedimien- } \\
\text { tos de organización. }\end{array}$ \\
\hline $\begin{array}{l}\text { Moulaert, MacCallum y Hillier (2013), Estensoro } \\
\text { (2015) }\end{array}$ & $\begin{array}{l}\text { La innovación social definida como un proceso de cambio, en } \\
\text { donde los actores se relacionan entre sí para apoyarse en su pro- } \\
\text { ceso de aprendizaje y la generación de nuevas capacidades. }\end{array}$ \\
\hline
\end{tabular}

Berzin, Pitt Catsouphes y Gaitan Rossi (2015),

Casado da Rocha y Calzada (2015), Mason,

Barraket, Friel, O’Rourke y Stenta (2015), Bulut

Autores que comparten el concepto de Phills et al. (2008).

et al. (2013)

Phillips et al. (2015), McCarthy, Whitelaw, Westley,

Crandall y Burnett (2014), Cajaiba Santana (2014), Autores que comparten el concepto de Mulgan et al. (2007).

Bonifacio (2014), Mason et al. (2015)

Fuente: elaboración de los autores a partir de la revisión de la literatura. 
La tendencia de 'aceptación' y utilización de los conceptos desarrollados por Mulgan et al. (2007) y Phills et al. (2008) da cuenta de que, a pesar de no ser unificado el concepto, empiezan a 'reconocerse' las mismas actividades como innovación social en los años posteriores.

Se observa además que hay dos tendencias marcadas en el concepto: la primera tiene que ver con las necesidades de la sociedad (ambientales, económicas, sociales, culturales, etc.) y cómo a través de la innovación se pueden solucionar dichas necesidades de forma diferente (Mulgan et al., 2007; Phills et al., 2008; Mumford, 2002; Marcy \& Mumford, 2007; Comisión Europea, 2010; Klein et al., 2012); y, por otro lado, la transformación de las redes

y la colaboración (Murray et al., 2009; (Moulaert, MacCallum, Mehmood et al., 2013; Van Dyck \& Van den Broeck, 2013; Moulaert, MacCallum \& Hillier, 2013; Estensoro, 2015) para conseguir objetivos comunes.

\subsubsection{Tendencias de investigación en innovación social}

En cuanto a las tendencias actuales de investigación, se observa que el estudio de la innovación social se aborda desde diversos niveles de análisis, como se muestra en la figura 6: general, macro, meso y micro.

Nivel general

A nivel general autores como Bonifacio (2014), Cajaiba Santana (2014), Olsson, Moore, Westley y McCarthy (2017), Edwards-Schachter y Wallace (2017) y Marques, Morgan y Richardson (2018) desarrollan conceptos ampliados de innovación social. Alijani, Luna, Castro Spila y Unceta (2016) y Milley, Szijarto, Svensson y Cousins (2018), por su parte, identifican impulsores y barreras de la innovación social. Además, es posible encontrar trabajos de asociación de la innovación social a los conceptos de innovación tecnológica (Bulut et al., 2013), innovación inclusiva (Tello Rozas, 2016), ética aplicada (Casado da Rocha \& Calzada, 2015), teoría institucional (Manning \& Roessler, 2014; Turker \& Altuntas Vural, 2017; Weisenfeld \& Hauerwaas, 2018; Windrum, Schartinger \& Waring, 2018) y gobernanza (Schicklinski, 2017). 
Nivel macro

A nivel macro se resalta la alta tendencia de estudiar la innovación social desde el enfoque ambiental (Sung \& Park, 2018; Moore et al., 2014; Tyfield, Ely \& Geall, 2015; Feola \& Nunes, 2014; McCarthy et al., 2014; Westley et al., 2013), económico (Arena, Bengo, Calderini \& Chiodo, 2018; Dwivedi \& Weerawardena, 2018; Newth, 2018; Cui, Pan, Newell \& Cui, 2017; Mongelli \& Rullani, 2017; Roundy \& Bonnal, 2017; Slimane \& Lamine, 2017; Alegre, Kislenko \& Berbegal Mirabent, 2017; Schröer \& Jäger, 2015) y social, relacionados con el desarrollo de las comunidades (Stott \& Tracey, 2018; Toivonen, 2016; Seyfang \& Longhurst, 2016; Lehtola \& Ståhle, 2014).

Dentro de los mayores aportes del estudio de la innovación social desde la perspectiva ambiental están la introducción de conceptos como grassroots innovations (Feola \& Nunes, 2014), socialecological system (SEs) (Moore et al., 2014), la planeación de innovaciones ecosistémicas (McCarthy et al., 2014) y la relación de los líderes con las innovaciones del enfoque socioambiental (Westley et al., 2013).

En las investigaciones desarrolladas bajo el enfoque económico se habla de la financiación (Arena et al., 2018) y de las transacciones (Slimane \& Lamine, 2017) de la innovación social. Se estudia la relación entre innovación social y el comercio electrónico (Cui et al., 2017), e innovación social y los emprendimientos sociales (Newth, 2018; Dwivedi \& Weerawardena, 2018; Lubberink, Blok, Van Ophem, Van der Velde \& Omta, 2018; Mongelli \& Rullani, 2017; Roundy \& Bonnal, 2017; Alegre et al., 2017; Sandeep \& Ravishankar, 2015, Marcy, 2015). También, se introduce el concepto de organizaciones híbridas (Schröer \& Jäger, 2015) y cómo se pueden combinar los beneficios económicos con la misión social de una organización.

Desde el enfoque social, que incluye el interés en el desarrollo de comunidades, según Sanzo Pérez et al. (2015), la innovación social se basa en su potencial de ser una fuente de bienestar para las comunidades y un medio para fortalecer el papel que desempeña la sociedad civil (Comisión Europea, 2010; Howaldt \& Schwarz, 2010). Así mismo, se está trabajando el concepto de social innovation community (SIC) y ciudades cosmopolitas innovadoras, reconocidas por compartir espacios culturales en espacios on/offlines (Toivonen, 2016), al igual que se evidencian estudios relacionados con los impactos en la sociedad civil de las innovaciones (Seyfang \& Longhurst, 2016; Lehtola \& Ståhle, 2014). 
Nivel meso

En el nivel meso se observa gran interés por el estudio de las ong y su relación con la innovación social (Shier \& Handy, 2015; Sanzo Pérez et al., 2015). White (2018) habla del valor de las empresas sociales y Weerawardena y Mort (2006) plantean que las empresas sin ánimo de lucro se consideran un "laboratorio" de las innovaciones sociales en términos de nuevos productos y los servicios prestados a los segmentos que no están cubiertos por el mercado o el Estado, nuevas organizaciones y nuevos procedimientos. De acuerdo con Bouchard (2012), existen tres razones que explican el potencial de las ong para el desarrollo de las innovaciones sociales: 1) la idoneidad sin fines de lucro para desarrollar servicios dirigidos al bien común debido a su restricción de no distribución; 2) su flexibilidad para responder rápidamente a las demandas específicas de la movilización de recursos de voluntarios; y 3) su capacidad para responder con prontitud a las necesidades sociales, ya que se rigen por las principales partes interesadas en estas necesidades. Conforme con Shier y Handy (2015), el principal motivo de estudio del papel de las ong es porque en ellas se desarrollan innovaciones, analizadas desde la teoría de las capacidades dinámicas y su influencia en la construcción de nuevas capacidades (Sanzo Pérez et al., 2015).

En este nivel también se aprecia el inicio de estudios en el sector salud (Mason et al., 2015), relacionados con el vínculo entre la innovación social y la promoción de la equidad en salud, en el sector público (Rana, Weerakkody, Dwivedi \& Piercy, 2014); y en el sector servicios (Gallouj, Rubalcaba, Toivonen \& Windrum, 2018), afines a la aplicación del concepto de innovación social, así como se nota el estudio de la relación entre la innovación social y el desarrollo de capacidades organizacionales (Urban \& Gaffurini, 2017, 2018) y los estudios asociados con incubadoras sociales (Nicolopoulou, Karataş-Özkan, Vas \& Nouman, 2017; Kautonen, Pugh \& Raunio, 2017).

Nivel micro

A este nivel se introduce el concepto de empresario moral (Antadze \& McGowan, 2017); además, se observa la tendencia a estudiar al innovador social y sus características (Estensoro, 2015; Sandeep \& Ravishankar, 2015; Berzin, Pitt Catsouphes \& Gaitan Rossi, 2015), y cómo a través de un estudio empírico se conoce la perspectiva de la innovación 
social desde la mirada de los líderes (Berzin et al., 2015), encontrando relación entre los líderes y el desarrollo de redes de colaboración (Estensoro, 2015).

De igual manera, se investiga la construcción de capital social (Bhatt \& Altinay, 2013), evidenciándose su aprovechamiento en las innovaciones sociales y su importancia para su desarrollo, al igual que los nuevos consumidores y su rol en esta nueva tendencia de innovación social (Jaeger Erben, Rückert John \& Schäfer, 2015).

\section{Discusiones y conclusiones}

La construcción conceptual de la innovación social se dio entre los años 2002 y 2010, mostrando dos grandes vertientes de investigación que han sido desarrolladas hasta la actualidad: la primera hace relación a las necesidades de la sociedad (ambientales, económicas, sociales, culturales, etc.) y cómo a través de la innovación se pueden solucionar dichas necesidades de forma diferente; y, por otro lado, la transformación de las redes y la colaboración para conseguir objetivos comunes.

En el desarrollo y consolidación de las investigaciones en innovación social se destaca la participación de Frances R. Westley y Per Olsson como los autores con mayor número de publicaciones; las revistas Ecology and Society y Technological Forecasting and Social Change como las que presentan el mayor número de artículos relacionados con innovación social; el Reino Unido y España como los países que más apoyan el desarrollo de estas investigaciones; el emprendimiento social como la palabra clave más relacionada con is; y la revisión de literatura, el método de casos y el análisis cualitativo como las metodologías más utilizadas en este tipo de investigaciones.

Actualmente se reconoce que aún no existe un consenso total del concepto de innovación social; no obstante, se observa una amplia aplicación de este desde los niveles general, macro, meso y micro, abordados desde distintos enfoques: ambiental, económico, social; diferentes sectores: público, privado, gubernamental, salud, servicios, sin ánimo de lucro; además del análisis del individuo con sus interacciones.

Basados en la revisión de literatura realizada, se puede observar que existen diferentes oportunidades de investigaciones en los distintos niveles: 
A nivel macro: existen pocas evidencias de investigaciones con innovación social y el aspecto económico, principalmente que puedan establecer claras diferencias y similitudes entre conceptos similares: responsabilidad social corporativa, emprendimiento social, negocios sociales, negocios inclusivos, valor compartido, etc. Así mismo, relacionados con evidencias desarrolladas en comunidades.

A nivel meso: en este ámbito la investigación está bastante incipiente, por lo que se pueden desarrollar proyectos aplicados a diferentes sectores, ya sea empresarial, privado, público u organizaciones no gubernamentales (Phillips et al., 2015, p. 26).

A nivel micro: oportunidades asociadas con el innovador social, características, perfiles, relación con el entorno; por otro lado, la interacción de los individuos con el entorno innovador, participación de los stakebolders, su papel, beneficios obtenidos, etc.

Igualmente, hay una brecha existente entre la innovación social y su estudio desde distintas teorías de análisis. En cuanto a las metodologías utilizadas, los estudios empíricos son los que presentan mayor debilidad, por lo que pocos modelos matemáticos han sido desarrollados. Por otro lado, el tema de los impactos sociales generados muestra un gran vacío de investigación y una necesidad altamente requerida para su satisfacción.

\section{Referencias}

Alegre, I., Kislenko, S., \& Berbegal-Mirabent, J. (2017). Organized chaos: mapping the definitions of social entrepreneurship. Journal of Social Entrepreneurship, 8(2), 248-264. https:// doi.org/10.1080/19420676.2017.1371631

Alijani, S., Luna, A., Castro-Spila, J., \& Unceta, A. (2016). Building capabilities through social innovation: implications for the economy and society. Finance and Economy for Society: Integrating Sustainability (Critical Studies on Corporate Responsibility, Governance and Sustainability), 11, 293-313.. https://doi.org/10.1108/S2043-905920160000011016

Antadze, N., \& McGowan, K. A. (2017). Moral entrepreneurship: thinking and acting at the landscape level to foster sustainability transitions. Environmental Innovation and Societal Transitions, 25, 1-13. https://doi.org/10.1016/J.EIST.2016.11.001 
Arcos Soto, C., Suárez Pineda, M., \& Zambrano Vargas, S. M. (2015). Procesos de innovación social (Is) como fuente de transformación social de comunidades rurales. Academia y Virtualidad, 8(2), 85-99.

Arena, M., Bengo, I., Calderini, M., \& Chiodo, V. (2018). Unlocking finance for social tech startups: is there a new opportunity space? Technological Forecasting and Social Change, 127, 154-165. https://doi.org/10.1016/J.TECHFORE.2017.05.035

Austin, J., Stevenson, H., \& Wei-Skillern, J. (2006). Social and commercial entrepreneurship: same, different, or both? Entrepreneurship Theory and Practice, 3O(1), 1-22. https://doi. org/10.1111/j.1540-6520.2006.00107.x

Berzin, S. C., Pitt Catsouphes, M., \& Gaitan Rossi, P. (2015). Defining our own future: human service leaders on social innovation. Human Service Organizations: Management, Leadership \& Governance, 39(5), 412-425. https://doi.org/10.1080/23303131.2015.1060914

Bhatt, P., \& Altinay, L. (2013). How social capital is leveraged in social innovations under resource constraints? Management Decision, 51(9), 1772-1792. https://doi.org/10.1108/ MD-01-2013-0041

Bonifacio, M. (2014). Social innovation: a novel policy stream or a policy compromise? An EU perspective. European Review, 22(1), 145-169.

Bouchard, M. J. (2012). Social innovation, an analytical grid for understanding the social economy: the example of the Québec housing sector. Service Business, 6(1), 47-59. https://doi. org/10.1007/s11628-011-0123-9

Bulut, C., Eren, H., \& Halac, D. S. (2013). Which one triggers the other? Technological or social innovation. Creativity Research Journal, 25(4), 436-445.

Cajaiba Santana, G. (2014). Social innovation: moving the field forward. A conceptual framework. Technological Forecasting and Social Change, 82, 42-51.

Casado da Rocha, A., \& Calzada, I. (2015). Demos-ethos: a framework to study the Icelandic and Basque cases through critical social innovation and applied ethics. Innovation: The European Journal of Social Science Research, 28(4), 425-442. https://doi.org/10.1080/1 3511610.2015 .1089472

Cheng, C. C. J., \& Krumwiede, D. (2017). What makes a manufacturing firm effective for service innovation? The role of intangible capital under strategic and environmental conditions. International Journal of Production Economics, 193, 113-122. 
Comisión Europea. (2010). Communication from the Commission to the European Parliament, the Council, the European Economic and Social Committee and the Committee of the Regions: Europe 2020 Flagship Initiative-Innovation Union. Bruselas.

Cui, M., Pan, S. L., Newell, S., \& Cui, L. (2017). Strategy, resource orchestration and e-commerce enabled social innovation in rural China. The Journal of Strategic Information Systems, 26(1), 3-21. https://doi.org/10.1016/J.JSIS.2016.10.001

Dwivedi, A., \& Weerawardena, J. (2018). Conceptualizing and operationalizing the social entrepreneurship construct. Journal of Business Research, 86, 32-40. https://doi.org/10.1016/J. JBUSRES.2018.01.053

Edwards-Schachter, M., \& Wallace, M. L. (2017). 'Shaken, but not stirred': sixty years of defining social innovation. Technological Forecasting and Social Change, 119, 64-79.

Estensoro, M. (2015). How can social innovation be facilitated? Experiences from an action research process in a local network. Systemic Practice and Action Research, 28(6), 527545. https://doi.org/10.1007/s11213-015-9347-2

Feola, G., \& Nunes, R. (2014). Success and failure of grassroots innovations for addressing climate change: the case of the transition movement. Global Environmental Change, 24, 232-250. https://doi.org/10.1016/J.GLOENVCHA.2013.11.011

Fichman, R. G., Dos Santos, B. L., \& Zheng, Z. E. (2014). Digital innovation as a fundamental and powerful concept in the information systems curriculum. MIS Quarterly, 38(2), 239-243.

Gallouj, F., Rubalcaba, L., Toivonen, M., \& Windrum, P. (2018). Understanding social innovation in services industries. Industry and Innovation, 25(6), 551-569. https://doi.org/10. 1080/13662716.2017.1419124

Howaldt, J., \& Schwarz, M. (2010). Social innovation. Concepts, research fields, and international trends. Dortmund: Sozialforschungstelle Dortmund.

Howaldt, J., \& Schwarz, M. (2011). Social innovation. Social challenges and future research fields. In S. Jeschke, I. Isenhardt, F. Hees \& S. Trantow (Eds.), Enabling innovation (pp. 203-223). Berlin-Heidelberg: Springer. https://doi.org/10.1007/978-3-642-24503-9_22

Jaeger Erben, M., Rückert John, J., \& Schäfer, M. (2015). Sustainable consumption through social innovation: a typology of innovations for sustainable consumption practices. Journal of Cleaner Production, 108, 784-798. https://doi.org/10.1016/J.JCLEPRO.2015.07.042

Kautonen, M., Pugh, R., \& Raunio, M. (2017). Transformation of regional innovation policies: from 'traditional' to 'next generation' models of incubation. European Planning Studies, 25(4), 620-637. https://doi.org/10.1080/09654313.2017.1281228

Klein, J. L. (2013). Introduction: social innovation at the crossroads between science, economy and society. In F. Moulaert, D. MacCallum, A. Mehmood \& A. Hamdouch, The international 
handbook on social innovation, collective action, social learning and transdisciplinary research.Cheltenham, uk: Edward Elgar Publishing.

Klein, J. L., Fontan, J. M., Harrisson, D., \& Lévesque, B. (2012). The Quebec system of social innovation: a focused analysis on the local development field. Finisterra-Revista Portuguesa de Geografia, (94), 9-28.

Lehtola, V. V., \& Ståhle, P. (2014). Societal innovation at the interface of the state and civil society. Innovation: The European Journal of Social Science Research, 27(2), 152-174.

Liu, X., Jiang, T., \& Ma, F. (2013). Collective dynamics in knowledge networks: emerging trends analysis. Journal of Informetrics, 7(2), 425-438.

Lubberink, R., Blok, V., Van Ophem, J., Van der Velde, G., \& Omta, O. (2018). Innovation for society: towards a typology of developing innovations by social entrepreneurs. Journal of Social Entrepreneurship, 9(1), 52-78. https://doi.org/10.1080/19420676.2017.1410212

Mair, J., \& Martí, I. (2006). Social entrepreneurship research: a source of explanation, prediction, and delight. Journal of World Business, 41(1), 36-44. https://doi.org/10.1016/J. JWB.2005.09.002

Manning, S., \& Roessler, D. (2014). The formation of cross-sector development partnerships: how bridging agents shape project agendas and longer-term alliances. Journal of Business Ethics, 123(3), 527-547. https://doi.org/10.1007/s10551-013-1853-5

Marcy, R. T. (2015). Breaking mental models as a form of creative destruction: the role of leader cognition in radical social innovations. The Leadership Quarterly, 26(3), 370-385. https://doi.org/10.1016/J.LEAQUA.2015.02.004

Marcy, R. T., \& Mumford, M. D. (2007). Social innovation: enhancing creative performance through causal analysis. Creativity Research Journal, 19(2-3), 123-140.

Marques, P., Morgan, K., \& Richardson, R. (2018). Social innovation in question: the theoretical and practical implications of a contested concept. Environment and Planning C: Politics and Space, 36(3), 496-512. https://doi.org/10.1177/2399654417717986

Martins, L. L., Rindova, V. P., \& Greenbaum, B. E. (2015). Unlocking the hidden value of concepts: a cognitive approach to business model innovation. Strategic Entrepreneurship Journal, 9(1), 99-117.

Mason, C., Barraket, J., Friel, S., O’Rourke, K., \& Stenta, C.-P. (2015). Social innovation for the promotion of health equity. Health Promotion International, 3O(suppl 2), ii116-ii125. https://doi.org/10.1093/heapro/dav076

McCarthy, D. D. P., Whitelaw, G. S., Westley, F. R., Crandall, D. D., \& Burnett, D. (2014). The Oak Ridges Moraine as a social innovation: strategic vision as a social-ecological interaction. Ecology and Society, 19(1), art48. https://doi.org/10.5751/ES-06212-190148 
Meissner, D., \& Kotsemir, M. (2016). Conceptualizing the innovation process towards the 'active innovation paradigm'. Trends and outlook. Journal of Innovation and Entrepreneurship, 5(1), 14.

Milley, P., Szijarto, B., Svensson, K., \& Cousins, J. B. (2018). The evaluation of social innovation: a review and integration of the current empirical knowledge base. Evaluation, 24(2), 237-258. https://doi.org/10.1177/1356389018763242

Mongelli, L., \& Rullani, F. (2017). Inequality and marginalisation: social innovation, social entrepreneurship and business model innovation. Industry and Innovation, 24(5), 446-467. https://doi.org/10.1080/13662716.2017.1295365

Moore, M.-L., Tjornbo, O., Enfors, E., Knapp, C., Hodbod, J., ... Biggs, D. (2014). Studying the complexity of change: toward an analytical framework for understanding deliberate social-ecological transformations. Ecology and Society, 19(4), art54. https://doi.org/10.5751/ ES-06966-190454

Moulaert, F., MacCallum, D., \& Hillier, J. (2013). Social innovation: intuition, precept, concept. In F. Moulaert, D. MacCallun, A. Mehmood \& A. Hamdouch (Eds.), The international handbook on social innovation: collective action, social learning and transdisciplinary research (pp. 13-24). Cheltenham, uk: Edward Elgar Publishing.

Moulaert, F., MacCallum, D., Mehmood, A., \& Hamdouch, A. (2013). General introduction: the return of social innovation as a scientific concept and a social practice. In F. Moulaert, D. MacCallun, A. Mehmood \& A. Hamdouch (Eds.), The international handbook on social innovations (pp. 1-6). Cheltenham, uk: Edward Elgar Publishing.

Moulaert, F., Martinelli, F., González, S., \& Swyngedouw, E. (2007). Introduction: social innovation and governance in European cities: urban development between path dependency and radical innovation. London: SAGE Publications.

Mulgan, G. (2006). The process of social innovation. Innovations: Technology, Governance, Globalization, 1(2), 145-162.

Mulgan, G., Tucker, S., Ali, R., \& Sanders, B. (2007). Social innovation: what it is, why it matters and how it can be accelerated.

Mumford, M. D. (2002). Social innovation: ten cases from Benjamin Franklin. Creativity Research Journal, 14(2), 253-266.

Murray, R., Caulier-Grice, J., \& Mulgan, G. (2009). Social venturing. Nesta.

Neumeier, S. (2012). Why do social innovations in rural development matter and should they be considered more seriously in rural development research? Proposal for a stronger focus on social innovations in rural development research. Sociologia Ruralis, 52(1), 48-69. 
Newth, J. (2018). "Hands-on” vs "arm's length" entrepreneurship research. International Journal of Entrepreneurial Behavior E Research, 24(3), 683-696. https://doi.org/10.1108/ IJEBR-09-2016-0315

Nicolopoulou, K., Karataş-Özkan, M., Vas, C., \& Nouman, M. (2017). An incubation perspective on social innovation: the London Hub. A social incubator. RED Management, 47(3), 368-384. https://doi.org/10.1111/radm.12179

Olsson, P., Moore, M.-L., Westley, F. R., \& McCarthy, D. D. P. (2017). The concept of the Anthropocene as a game-changer: a new context for social innovation and transformations to sustainability. Ecology and Society, 22(2), art31. https://doi.org/10.5751/ES-09310220231

Phillips, W., Lee, H., Ghobadian, A., O'Regan, N., \& James, P. (2015). Social innovation and social entrepreneurship: a systematic review. Group \& Organization Management, 40(3), 428-461.

Phills, J. A., Deiglmeier, K., \& Miller, D. T. (2008). Rediscovering social innovation. Stanford Social Innovation Review, 6(4), 34-43.

Rana, N. P., Weerakkody, V., Dwivedi, Y. K., \& Piercy, N. C. (2014). Profiling existing research on social innovation in the public sector. Information Systems Management, 31(3), 259-273.

Rao Nicholson, R., Vorley, T., \& Khan, Z. (2017). Social innovation in emerging economies: a national systems of innovation based approach. Technological Forecasting and Social Change, 121, 228-237. https://doi.org/10.1016/J.TECHFORE.2017.03.013

Robledo Giraldo, S., Duque Méndez, N. D., \& Zuluaga Giraldo, J. I. (2013). Difusión de productos a través de redes sociales: una revisión bibliográfica utilizando la teoría de grafos. Respuestas, 18(2), 28-42.

Robledo, S., Osorio, G., \& López, C. (2014). Networking en pequeña empresa: una revisión bibliográfica utilizando la teoria de grafos. Revista Vínculos, 11(2), 6-16.

Roundy, P. T., \& Bonnal, M. (2017). The singularity of social entrepreneurship: untangling its uniqueness and market function. The Journal of Entrepreneurship, 26(2), 137-162. https:// doi.org/10.1177/0971355717708068

Sandeep, M. S., \& Ravishankar, M. N. (2015). Social innovations in outsourcing: an empirical investigation of impact sourcing companies in India. The Journal of Strategic Information Systems, 24(4), 270-288. https://doi.org/10.1016/J.JsIs.2015.09.002

Sanzo Pérez, M. J., Álvarez González, L. I., \& Rey García, M. (2015). How to encourage social innovations: a resource-based approach. The Service Industries Journal, 35(7-8), 430-447.

Schicklinski, J. (2017). The governance of green urban spaces in the EU: social innovation and civil society. London: Routledge. Available from https://www.routledge.com/The- 
Governance-of-Urban-Green-Spaces-in-the-EU-Social-innovation-and-civil/Schicklinski/p/ book/9781138223752

Schröer, A., \& Jäger, U. (2015). Beyond balancing? International Studies of Management $\mathcal{E}$ Organization, 45(3), 259-281. https://doi.org/10.1080/00208825.2015.1006032

Seyfang, G., \& Longhurst, N. (2016). What influences the diffusion of grassroots innovations for sustainability? Investigating community currency niches. Technology Analysis \& Strategic Management, 28(1), 1-23. https://doi.org/10.1080/09537325.2015.1063603

Seyfang, G., \& Smith, A. (2007). Grassroots innovations for sustainable development: towards a new research and policy agenda. Environmental Politics, 16(4), 584-603. https://doi. org/10.1080/09644010701419121

Shier, M. L., \& Handy, F. (2015). Social change efforts of direct service nonprofits: the role of funding and collaborations in shaping social innovations. Human Service Organizations: Management, Leadership \& Governance, 39(1), 6-24.

Silva, G. M., Styles, C., \& Lages, L. F. (2017). Breakthrough innovation in international business: the impact of tech-innovation and market-innovation on performance. International Business Review, 26(2), 391-404.

Slimane, K. B., \& Lamine, W. (2017). A transaction-based approach to social innovation. The International Journal of Entrepreneurship and Innovation, 18(4), 231-242. https://doi. org/10.1177/1465750317741879

Souto, J. E. (2015). Business model innovation and business concept innovation as the context of incremental innovation and radical innovation. Tourism Management, 51, 142-155.

Stephan, A., Schmidt, T. S., Bening, C. R., \& Hoffmann, V. H. (2017). The sectoral configuration of technological innovation systems: patterns of knowledge development and diffusion in the lithium-ion battery technology in Japan. Research Policy, 46(4), 709-723.

Stott, N., \& Tracey, P. (2018). Organizing and innovating in poor places. Innovation, 20(1), 1-17. https://doi.org/10.1080/14479338.2017.1358093

Sung, B., \& Park, S.-D. (2018). Who drives the transition to a renewable-energy economy? Multi-actor perspective on social innovation. Sustainability, 1O(2), 1-32. Available from https://ideas.repec.org/a/gam/jsusta/v10y2018i2p448-d130958.html

Tello Rozas, S. (2016). Inclusive innovations through social and solidarity economy initiatives: a process analysis of a Peruvian case study. Voluntas: International Journal of Voluntary and Nonprofit Organizations, 27(1), 61-85. https://doi.org/10.1007/s11266-015-9606-y

Toivonen, T. (2016). What is the social innovation community? Conceptualizing an emergent collaborative organization. Journal of Social Entrepreneurship, 7(1), 49-73. https://doi.or $\mathrm{g} / 10.1080 / 19420676.2014 .997779$ 
Turker, D., \& Altuntas Vural, C. (2017). Embedding social innovation process into the institutional context: voids or supports. Technological Forecasting and Social Change, 119, 98-113. https://doi.org/10.1016/J.TECHFORE.2017.03.019

Tyfield, D., Ely, A., \& Geall, S. (2015). Low carbon innovation in China: from overlooked opportunities and challenges to transitions in power relations and practices. Sustainable Development, 23(4), 206-216. https://doi.org/10.1002/sd.1588

Urban, B., \& Gaffurini, E. (2017). Organisational learning capabilities as determinants of social innovation: an empirical study in South Africa. SAJournal of Human Resource Management, 15(0), 10. https://doi.org/10.4102/sajhrm.v15i0.857

Urban, B., \& Gaffurini, E. (2018). Social enterprises and organizational learning in South Africa. Journal of Entrepreneurship in Emerging Economies, 1O(1), 117-133. https://doi. org/10.1108/JEEE-02-2017-0010

Van der Have, R. P., \& Rubalcaba, L. (2016). Social innovation research: an emerging area of innovation studies? Research Policy, 45(9), 1923-1935.

Van Dyck, B., \& Van den Broeck, P. (2013). Social innovation: a territorial process. In The international handbook on social innovation: collective action, social learning and transdisciplinary research (pp. 131-141). Cheltenham, uk: Edward Elgar. http://dx.doi. org/10.4337/9781849809993.00021

Weerawardena, J., \& Mort, G. S. (2006). Investigating social entrepreneurship: a multidimensional model.Journal of World Business, 41(1), 21-35. https://doi.org/10.1016/J.JWв.2005.09.001

Weisenfeld, U., \& Hauerwaas, A. (2018). Adopters build bridges: changing the institutional logic for more sustainable cities. From action to workset to practice. Research Policy, 47(5), 911-923. https://doi.org/10.1016/J.RESPOL.2018.02.015

Westley, F. R., Tjornbo, O., Schultz, L., Olsson, P., Folke, C., ... Bodin, Ö. (2013). A theory of transformative agency in linked social-ecological systems. Ecology and Society, 18(3), art27. https://doi.org/10.5751/ES-05072-180327

White, L. (2018). A Cook's tour: towards a framework for measuring the social impact of social purpose organisations. European Journal of Operational Research, 268(3), 784-797. https://doi.org/10.1016/J.EJOR.2017.06.015

Windrum, P., Schartinger, D., \& Waring, J. (2018). Co-creation of social innovations and new professional institutions: diffusion of therapeutic patient education (TPE) for diabetes in Austria. Industry and Innovation, 25(6), 570-593. https://doi.org/10.1080/13662716.20 17.1295363 
Witkamp, M. J., Raven, R. P. J. M., \& Royakkers, L. M. M. (2011). Strategic niche management of social innovations: the case of social entrepreneurship. Technology Analysis \& Strategic Management, 23(6), 667-681. https://doi.org/10.1080/09537325.2011.585035 